\title{
Density of Tautologies in Logics with One Variable
}

\author{
László Aszalós* and Tamás Herendi*
}

\begin{abstract}
In the present paper we estimate the ratio of the number of tautologies and the number of formulae of length $n$ by determining the asymptotic density of tautologies in different kinds of logics with one variable. The logics under consideration are the ones with a single connective (nand or nor); negation with a connective (disjunction or conjunction); and several connectives.
\end{abstract}

Keywords: tautologies, density, generating functions

\section{Introduction}

One of the authors developed a computer aided education software of introductory logic, and he needed to generate formulae randomly. Naturally the following question has arisen: What percent of the randomly generated formulae are tautologies (logical law) or antilogies (contradiction)? We intend to answer this question for several logics in the present paper.

Traditionally this answer is a number expressing the ratio of favourable cases (tautologies) to the whole number of the cases possible (formulae). Since there are infinitely many tautologies, the ratio became $\infty / \infty$, which is not suitable for us. To find a reasonable answer one have to use some approximation. At first we made some experiments, we wrote programs to list the first ten million formulae (ordered by their length) and to count the tautologies among them. The test showed that the quotient of the numbers of tautologies and formulae may have a limit. Next we calculated the numbers of different kinds of formulae up to the first $10^{100}$ formulae. We obtained similar results. Later we realized that Marek Zaionc gave the exact value of the asymptotic limit for some logics [10]. He, together with his collegues and students, examined purely implicational [6], intuitionistic [7, 5] and modal [3, 4] logics with a few propositional variables. Moreover he dealt with the case of several propositional variables [11]. Harris studied the $\mathrm{k}$-cnf case [2]. In the present paper we provide limits for logics with one propositional variable. Our main tool for the observation of asymptotics of sequences is generating function [9]. The exposition of the method are nicely presented in the articles cited before, e.g. in [10]. Although we used the Zaionc's method, our set of logical connectives are not discussed yet.

\footnotetext{
*Faculty of Informatics, University of Debrecen, Egyetem tér 1, H4032 Debrecen, Hungary, E-mail: \{Laszlo.Aszalos, herendi\}@inf .unideb.hu
} 


\section{Definitions and preliminary results}

In this section we introduce the concept of generating functions, and we give some basic properties of them.

Definition 1. A complex function $f$ is said to be analytic on a region $R$ if it is differentiable at every point in $R$. It is also called regular, if it is analytic and single valued in $R$.

Definition 2. Suppose that the complex function $f$ is analytic on a region $R$. Then a point $z$ on the boundary is called regular if the extension of $f$ on an open region containing $R \cup\{z\}$ is analytic. The point $z$ is called singular, if it is not regular.

Remark 1. The complex function $f(z)=\sqrt{z}$ has the only singularity at $z_{0}=0$.

Remark 2. Let $p(z)$ be a polynomial of degree $d$ with not necessarily different roots $z_{1}, \ldots, z_{d}$. Since $p$ is analytic on the whole complex plane, the function $\sqrt{p(z)}$ may have singularity only at the points, where $p(z)=0$, i.e. at $z_{1}, \ldots, z_{d}$.

Definition 3. Let $u_{n}$ be a sequence of real numbers. The formal power series $U(x)=\sum_{n=0}^{\infty} u_{n} x^{n}$ is called the generating function of the sequence $u_{n}$.

Remark 3. Let $u_{n}, v_{n}$ and $w_{n}$ be sequences of numbers with generating functions $U(x), V(x)$ and $W(x)$, respectively, and let $\alpha$ be a real number. Then the following properties hold:

1. If $v_{n}=u_{n+1}$ for $n \geq 0$ then $U(x)=x V(x)+u_{0}$.

2. If $w_{n}=\sum_{i=0}^{n} u_{i} v_{n-i}$ for $n \geq 0$ then $W(x)=U(x) V(x)$.

3. If $w_{n}=u_{n}+v_{n}$ for $n \geq 0$ then $W(x)=U(x)+V(x)$.

4. If $v_{n}=\alpha \cdot u_{n}$ for $n \geq 0$ then $V(x)=\alpha \cdot U(x)$.

Theorem 1. Let $u_{n}$ and $v_{n}$ be two sequences of real numbers with generating functions $U(x)$ and $V(x)$, respectively. Suppose that both $U(x)$ and $V(x)$ have singularity in $x_{0}$ and they have no other singularities in the circle $|x| \leq\left|x_{0}\right|$. If

$$
U\left(x_{0} \cdot x\right)=\sum_{n=0}^{\infty} \hat{u}_{n}(1-x)^{\frac{n}{2}} \quad \text { and } \quad V\left(x_{0} \cdot x\right)=\sum_{n=0}^{\infty} \hat{v}_{n}(1-x)^{\frac{n}{2}}
$$

and $\hat{v}_{1} \neq 0$ then the limit $u_{n} / v_{n}$ exists and $\lim _{n \rightarrow \infty} u_{n} / v_{n}=\hat{u}_{1} / \hat{v}_{1}$.

Proof. In the proof we follow the arguments of Theorem 21 of [10].

By the assumed properties of $U(x)$ and $V(x)$ we may define $\bar{U}(x)=U\left(x_{0} \cdot x\right)$ and $\bar{V}(x)=V\left(x_{0} \cdot x\right)$ such that $\bar{U}(x)$ and $\bar{V}(x)$ are regular for $|x|<1$ and have the only singularity in $x=1$ on the unit circle. Hence $\bar{U}(x)=\sum_{n=0}^{\infty} \hat{u}_{n}(1-x)^{\frac{n}{2}}$ and $\bar{V}(x)=\sum_{n=0}^{\infty} \hat{v}_{n}(1-x)^{\frac{n}{2}}$. Let $\bar{u}_{n}=u_{n} / x_{0}^{n}$ and $\bar{v}_{n}=v_{n} / x_{0}^{n}$ be two sequences. Then $\bar{U}(x)$ and $\bar{V}(x)$ are the generating functions of $\bar{u}_{n}$ and $\bar{v}_{n}$, respectively. Clearly

$$
\lim _{n \rightarrow \infty} \frac{\bar{u}_{n}}{\bar{v}_{n}}=\lim _{n \rightarrow \infty} \frac{u_{n} / x_{0}^{n}}{v_{n} / x_{0}^{n}}=\lim _{n \rightarrow \infty} \frac{u_{n}}{v_{n}} .
$$


By Theorem 8.4 of [8] the asymptotic expansion of the coefficient of $x^{n}$ in $\bar{U}(x)$ and $\bar{V}(x)$ are

$$
\hat{u}_{1}\left(\begin{array}{c}
1 / 2 \\
n
\end{array}\right)(-1)^{n}+O\left(n^{-2}\right) \quad \text { and } \quad \hat{v}_{1}\left(\begin{array}{c}
1 / 2 \\
n
\end{array}\right)(-1)^{n}+O\left(n^{-2}\right),
$$

respectively. Hence

$$
\lim _{n \rightarrow \infty} \frac{\bar{u}_{n}}{\bar{v}_{n}}=\lim _{n \rightarrow \infty} \frac{\hat{u}_{1}\left(\begin{array}{c}
1 / 2 \\
n
\end{array}\right)(-1)^{n}+O\left(n^{-2}\right)}{\hat{v}_{1}\left(\begin{array}{c}
1 / 2 \\
n
\end{array}\right)(-1)^{n}+O\left(n^{-2}\right)}
$$

Since $\lim _{n \rightarrow \infty}\left(\begin{array}{c}1 / 2 \\ n\end{array}\right)(-1)^{n}=-\infty$, thus

$$
\lim _{n \rightarrow \infty} \frac{\hat{u}_{1}\left(\begin{array}{c}
1 / 2 \\
n
\end{array}\right)(-1)^{n}+O\left(n^{-2}\right)}{\hat{v}_{1}\left(\begin{array}{c}
1 / 2 \\
n
\end{array}\right)(-1)^{n}+O\left(n^{-2}\right)}=\lim _{n \rightarrow \infty} \frac{\hat{u}_{1}\left(\begin{array}{c}
1 / 2 \\
n
\end{array}\right)(-1)^{n}}{\hat{v}_{1}\left(\begin{array}{c}
1 / 2 \\
n
\end{array}\right)(-1)^{n}}=\lim _{n \rightarrow \infty} \frac{\hat{u}_{1}}{\hat{v}_{1}}=\frac{\hat{u}_{1}}{\hat{v}_{1}} .
$$

Combining this result with (1), we obtain the statement of the theorem.

In the present paper we use the following method for the computation of the relative frequency of the tautologies:

1. We determine the generating function $T$ of the number of tautologies and the generating function $S$ of the number of formulae.

2. We calculate the minimal singularities of $T$ and $S$, and we show that they are equal. We denote this common singularity by $x_{0}$.

3. We compute the expansion of $T\left(x_{0} \cdot x\right)$ and $S\left(x_{0} \cdot x\right)$ around the common singularity.

4. The limit of the relative frequency of the tautologies is $\hat{t}_{1} / \hat{s}_{1}$.

\section{Logical foundations}

Definition 4. Let $\Gamma$ be a nonempty set of unary (negation: $\neg$ ) and binary operators (disjunction: $\vee$, conjunction: $\wedge$, nand: $\uparrow$, nor: $\downarrow$, implication: $\supset$ and equivalence: $\equiv)$ and let $p$ be a unique propositional letter. The set of formulae of the logic of $\Gamma$ is the smallest set $\mathcal{F}^{\Gamma}$ for which

- $p \in \mathcal{F}^{\Gamma}$

- If $\neg \in \Gamma$ and $\varphi \in \mathcal{F}^{\Gamma}$, then $\neg \varphi \in \mathcal{F}^{\Gamma}$.

- If $\triangle \in \Gamma$ and $\varphi, \psi \in \mathcal{F}^{\Gamma}$, then $(\varphi \triangle \psi) \in \mathcal{F}^{\Gamma}$.

The length of a formula is defined as follows: $l(p)=1, l(\neg \varphi)=l(\varphi)+1$ and $l(\varphi \triangle \psi))=l(\varphi)+l(\psi)+1$. 
The length of a formula is equal to the numbers of its characters excluding the parentheses (or exactly the number of its characters in polish notation).

We say that formulae $\varphi$ and $\psi$ are equivalent, if the formula $(\neg \varphi \wedge \neg \psi) \vee(\varphi \wedge \psi)$ is tautology. We have only one propositional letter $p$, which may have two different truth-values. Since the value of a formula can be true or false in both cases, we have four different kinds of formulae. These equivalence classes based on the equivalence defined before are denoted by their representatives: $p, \neg p, \top$ and $\perp$. These sets are the classes of those formulae which are equivalent with $p, \neg p$, tautologies and antilogies, respectively. We will call the members of the equivalence class of $p$ pformulae, and the members of the equivalence class of $\neg p$ n-formulae. Denote the numbers of the tautologies and antilogies, p-formulae and n-formulae of length $n-1$ with $\top_{n}^{\Gamma}, \perp_{n}^{\Gamma}, P_{n}^{\Gamma}$ and $N_{n}^{\Gamma}$, respectively. If there is no danger of confusion, we omit the $\Gamma$ from notations.

If we negate an $n$-formula, we get a p-formula, if we connect with a conjunction an n-formula and a p-formula we get an antilogy, etc. We summarize this in the following tables:

\begin{tabular}{c|ccc|cccccc|cccc} 
& $\neg$ & $\wedge$ & $\perp$ & $p$ & $\neg p$ & $\top$ & & $\vee$ & $\perp$ & $p$ & $\neg p$ & $\top$ \\
\hline$\perp$ & $\top$ & & $\perp$ & $\perp$ & $\perp$ & $\perp$ & $\perp$ & & $\perp$ & $\perp$ & $p$ & $\neg p$ & $\top$ \\
$p$ & $\neg p$ & $p$ & $\perp$ & $p$ & $\perp$ & $p$ & $p$ & $p$ & $p$ & $\top$ & $\top$ \\
$\neg p$ & $p$ & & $\neg p$ & $\perp$ & $\perp$ & $\neg p$ & $\neg p$ & & $\neg p$ & $\neg p$ & $\top$ & $\neg p$ & $\top$ \\
$\top$ & $\perp$ & $\top$ & $\perp$ & $p$ & $\neg p$ & $\top$ & & $\top$ & $\top$ & $\top$ & $\top$ & $\top$ \\
& $\uparrow$ & $\perp$ & $p$ & $\neg p$ & $\top$ & & $\downarrow$ & $\perp$ & $p$ & $\neg p$ & $\top$ \\
\hline & $\perp$ & $\top$ & $\top$ & $\top$ & $\top$ & & $\perp$ & $\top$ & $\neg p$ & $p$ & $\perp$ \\
& $\neg$ & $\top$ & $\neg p$ & $\top$ & $\neg p$ & $p$ & $\neg p$ & $\neg p$ & $\perp$ & $\perp$ & \\
& $\neg p$ & $\top$ & $\top$ & $p$ & $p$ & & $\neg p$ & $p$ & $\perp$ & $p$ & $\perp$ & \\
& $\top$ & $\top$ & $\neg p$ & $p$ & $\perp$ & $\top$ & $\perp$ & $\perp$ & $\perp$ & $\perp$ &
\end{tabular}

Theorem 2. $P_{n}^{\{\neg, \vee\}}=P_{n}^{\{\neg, \wedge\}}, N_{n}^{\{\neg, \vee\}}=N_{n}^{\{\neg, \wedge\}}, \top_{n}^{\{\neg, \vee\}}=\perp_{n}^{\{\neg, \wedge\}}$ and $\perp_{n}^{\{\neg, \vee\}}=\top_{n}^{\{\neg, \wedge\}}$.

Remark 4. These equivalence classes form a complete lattice which should remind the reader to the Belnap's 4 valued system [1], but in our case there are no two natural ordering. Here the operations join and meet are the operation or and and, respectively. Fortunately we have only four equivalence classes and we can formulate the operation of $\uparrow$ (nand) as the join of the complementer set of the set of the operands, e.g. $\quad p \uparrow \neg p=\top \vee \perp$, where the operands are $p$ and $\neg p$, and their complementer set is $\{\top, \perp\}$. Similar statement holds for $\downarrow$ (nor $)$ : it is the meet of the complementer set of the set of the operands.

Proof. We prove Theorem 2 by induction on $n$. In the logic of negation and conjunction $P_{0}^{\{\neg, \wedge\}}=N_{0}^{\{\neg, \wedge\}}=\perp_{0}^{\{\neg, \wedge\}}=\top_{0}^{\{\neg, \wedge\}}=0, P_{1}^{\{\neg, \wedge\}}=N_{1}^{\{\neg, \wedge\}}=\perp_{1}^{\{\neg, \wedge\}}=$ $\top_{1}^{\{\neg, \wedge\}}=0, N_{2}^{\{\neg, \wedge\}}=\perp_{2}^{\{\neg, \wedge\}}=\top_{2}^{\{\neg, \wedge\}}=0$ and $P_{2}^{\{\neg, \wedge\}}=1$. Similar facts hold for $\top_{n}^{\{\neg, \vee\}}, \perp_{n}^{\{\neg, \vee\}}, P_{n}^{\{\neg, \vee\}}$ and $N_{n}^{\{\neg, \vee\}}$ if $n \leq 2$. Hence the statement is true for $n=0,1$ and 2 . 
According to (2) the following relations hold:

$$
\begin{aligned}
& P_{n}^{\{\neg, \vee\}}=N_{n-1}^{\{\neg, \vee\}}+\sum_{i=1}^{n-1} P_{i}^{\{\neg, \vee\}}\left(2 \perp_{n-i}^{\{\neg, \vee\}}+P_{n-i}^{\{\neg, \vee\}}\right) \\
& P_{n}^{\{\neg, \wedge\}}=N_{n-1}^{\{\neg, \wedge\}}+\sum_{i=1}^{n-1} P_{i}^{\{\neg, \wedge\}}\left(2 \top_{n-i}^{\{\neg, \wedge\}}+P_{n-i}^{\{\neg, \wedge\}}\right) \\
& N_{n}^{\{\neg, \vee\}}=P_{n-1}^{\{\neg, \vee\}}+\sum_{i=1}^{n-1} N_{i}^{\{\neg, \vee\}}\left(2 \perp{ }_{n-i}^{\{\neg, \vee\}}+N_{n-i}^{\{\neg, \vee\}}\right) \\
& N_{n}^{\{\neg, \wedge\}}=P_{n-1}^{\{\neg, \wedge\}}+\sum_{i=1}^{n-1} N_{i}^{\{\neg, \wedge\}}\left(2 \top_{n-i}^{\{\neg, \wedge\}}+N_{n-i}^{\{\neg, \wedge\}}\right) \\
& \perp_{n}^{\{\neg, \vee\}}=\top_{n-1}^{\{\neg, \vee\}}+\sum_{i=1}^{n-1} \perp_{i}^{\{\neg, \vee\}} \perp_{n-i}^{\{\neg, \vee\}} \\
& \top_{n}^{\{\neg, \wedge\}}=\perp_{n-1}^{\{\neg, \wedge\}}+\sum_{i=1}^{n-1} \top_{i}^{\{\neg, \wedge\}} \top_{n-i}^{\{\neg, \wedge\}} \\
& \underset{n}{\{\neg, \vee\}}=\perp_{n-1}^{\{\neg, \vee\}}+\sum_{i=1}^{n-1} \top_{i}^{\{\neg, \vee\}}\left(2 \perp_{n-i}^{\{\neg, \vee\}}+2 P_{n-i}^{\{\neg, \vee\}}+2 N_{n-i}^{\{\neg, \vee\}}+\top_{n-i}^{\{\neg, \vee\}}\right) \\
& +\sum_{i=1}^{n-1} P_{i}^{\{\neg, \vee\}} N_{n-i}^{\{\neg, \vee\}} \\
& \perp_{n}^{\{\neg, \wedge\}}=\top_{n-1}^{\{\neg, \wedge\}}+\sum_{i=1}^{n-1} \perp_{i}^{\{\neg, \wedge\}}\left(2 \top_{n-i}^{\{\neg, \wedge\}}+2 P_{n-i}^{\{\neg, \wedge\}}+2 N_{n-i}^{\{\neg, \wedge\}}+\perp_{n-i}^{\{\neg, \wedge\}}\right) \\
& +\sum_{i=1}^{n-1} P_{i}^{\{\neg, \wedge\}} N_{n-i}^{\{\neg, \wedge\}}
\end{aligned}
$$

Assume that our hypothesis hold for all numbers less than $n$. We want to prove it for $n$. Let us examine the equations of $P_{n}^{\{\neg, \wedge\}}$ and $P_{n}^{\{\neg, \vee\}}$ ! By the hypothesis $N_{n-1}^{\{\neg, \wedge\}}=N_{n-1}^{\{\neg, \vee\}}, P_{i}^{\{\neg, \wedge\}}=P_{i}^{\{\neg, \vee\}}$ and $\top_{n-i}^{\{\neg, \wedge\}}=\perp_{n-i}^{\{\neg, \vee\}}$ for all $i<n$. The right sides of equations are equal, hence $P_{n}^{\{\neg, \wedge\}}$ and $P_{n}^{\{\neg, \vee\}}$ are equal, too. The verification of the equalities of the other pairs is left to the reader.

A similar theorem holds for the logic of nand and the logic of nor:

Theorem 3. $P_{n}^{\{\downarrow\}}=P_{n}^{\{\uparrow\}}, N_{n}^{\{\downarrow\}}=N_{n}^{\{\uparrow\}}, \top_{n}^{\{\downarrow\}}=\perp_{n}^{\{\uparrow\}}$ and $\perp_{n}^{\{\downarrow\}}=\top_{n}^{\{\uparrow\}}$. 
Proof. By (3) we have:

$$
\begin{aligned}
P_{n}^{\{\uparrow\}} & =\sum_{i=1}^{n-1} N_{i}^{\{\uparrow\}}\left(2 \top_{n-i}^{\{\uparrow\}}+N_{n-i}^{\{\uparrow\}}\right) \\
P_{n}^{\{\downarrow\}} & =\sum_{i=1}^{n-1} N_{i}^{\{\downarrow\}}\left(2 \perp_{n-i}^{\{\downarrow\}}+N_{n-i}^{\{\downarrow\}}\right) \\
N_{n}^{\{\uparrow\}} & =\sum_{i=1}^{n-1} P_{i}^{\{\uparrow\}}\left(2 \top_{n-i}^{\{\uparrow\}}+P_{n-i}^{\{\uparrow\}}\right) \\
N_{n}^{\{\downarrow\}} & =\sum_{i=1}^{n-1} P_{i}^{\{\downarrow\}}\left(2 \perp_{n-i}^{\{\downarrow\}}+P_{n-i}^{\{\downarrow\}}\right) \\
\perp_{n}^{\{\uparrow\}} & =\sum_{i=1}^{n-1} T_{i}^{\{\uparrow\}} T_{n-i}^{\{\uparrow\}} \\
T_{n}^{\{\downarrow\}} & =\sum_{i=1}^{n-1} \perp_{i}^{\{\downarrow\}} \perp_{n-i}^{\{\downarrow\}} \\
\top_{n}^{\{\uparrow\}} & =\sum_{i=1}^{n-1} \perp_{i}^{\{\uparrow\}}\left(2 \top_{n-i}^{\{\uparrow\}}+2 P_{n-i}^{\{\uparrow\}}+2 N_{n-i}^{\{\uparrow\}}+\perp_{n-i}^{\{\uparrow\}}\right) \\
\perp_{n}^{\{\downarrow\}} & =\sum_{i=1}^{n-1} T_{i}^{\{\downarrow\}}\left(2 \perp_{n-i}^{\{\downarrow\}}+2 P_{n-i}^{\{\downarrow\}}+2 N_{n-i}^{\{\downarrow\}}+T_{n-i}^{\{\downarrow\}}\right)
\end{aligned}
$$

The statement follows by induction on $n$.

Theorem 4. Each formula in $\mathcal{F}^{\{\uparrow\}}$ have odd length.

Proof. We prove the statement by induction on the length of formulae of $\mathcal{F}\{\uparrow\}$. The only formula of length 1 is $p$. Hence we can assume the statement for all formula of length less than or equal to $n$, where $n \geq 1$. Take a formula of length $n+1$, which can be only a formula of the form $A \uparrow B$, where the length of $A$ and $B$ are less than $n$, therefore both of them are odd-length formulae. By definition, the length of $A \uparrow B$ will be odd again, which concludes the proof.

To simplify the calculations we construct four sets of equivalence classes: $a=$ $\{\perp, p\}, b=\{\neg p, \top\}, c=\{\perp, \neg p\}$ and $d=\{p, \top\}$. Denote by $A_{n}^{\Gamma}, B_{n}^{\Gamma}, C_{n}^{\Gamma}$ and by $D_{n}^{\Gamma}$ the numbers of formulae length $n-1$ belonging to the sets $a, b, c$ and $d$, respectively. It is obvious, that $A_{n}^{\Gamma}=\perp_{n}^{\Gamma}+P_{n}^{\Gamma}, B_{n}^{\Gamma}=\top_{n}^{\Gamma}+N_{n}^{\Gamma}, C_{n}^{\Gamma}=\perp_{n}^{\Gamma}+N_{n}^{\Gamma}$ and $D_{n}^{\Gamma}=\top_{n}^{\Gamma}+P_{n}^{\Gamma}$.

We leave it to the reader to check that if each operand is from the same set then the results of the operation also belong to the same set. We summarize this 
in the following tables:

\begin{tabular}{|c|c|c|c|c|c|c|c|c|c|c|c|c|c|c|}
\hline & $\neg$ & & $\neg$ & $\wedge$ & $\mathrm{a}$ & b & $\wedge$ & $\mathrm{c}$ & $\mathrm{d}$ & $\uparrow$ & a & $\mathrm{b}$ & $\uparrow$ & $\mathrm{c}$ \\
\hline $\mathrm{a}$ & $\mathrm{b}$ & $\mathrm{c}$ & $\mathrm{d}$ & $\mathrm{a}$ & $\mathrm{a}$ & $\mathrm{a}$ & $\mathrm{c}$ & $\mathrm{c}$ & $\mathrm{c}$ & $\mathrm{a}$ & $\mathrm{b}$ & $\mathrm{b}$ & $\mathrm{c}$ & $\mathrm{d}$ \\
\hline b & $\mathrm{a}$ & $\mathrm{d}$ & $\mathrm{c}$ & b & $\mathrm{a}$ & $\mathrm{b}$ & $\mathrm{d}$ & $c$ & $\mathrm{~d}$ & $\mathrm{~b}$ & b & $\mathrm{a}$ & $\mathrm{d}$ & $\mathrm{d}$ \\
\hline
\end{tabular}

\section{Generating functions}

Let $S_{n}^{\Gamma}$ denote the number of formulae of length $n-1$ of the logic corresponding to $\Gamma$ if $n>1$, and let $S_{1}=S_{0}=0$.

Lemma 1.

$$
S_{n}^{\{\neg, \wedge\}}=S_{n-1}^{\{\neg, \wedge\}}+\sum_{i=1}^{n-1} S_{i}^{\{\neg, \wedge\}} S_{n-i}^{\{\neg, \wedge\}} \text { for } n>2
$$

Proof. A formula of length $n-1$ can be either a negation of a formula of length $n-2$ or can be a formula of the kind of $\varphi \Delta \psi$, where $\varphi$ and $\psi$ has length $i-1$ and $n-1-i$, respectively.

Let

$$
S(x)=\sum_{n=0}^{\infty} S_{n}^{\{\neg, \wedge\}} x^{n}
$$

be the generating function of $S_{n}^{\{\neg, \wedge\}}$. For the sake of brevity the generating function of $S_{n}^{\{\neg, \wedge\}}$ will be denoted by $S$.

Lemma 2.

$$
S=\frac{1-x-\sqrt{1-2 x-3 x^{2}}}{2}
$$

Proof. By (5) and the properties of generating functions we may write

$$
S=x S+S^{2}+x^{2} .
$$

We had to add $x^{2}$ to the right side, because $S_{2}^{\{\neg, \wedge\}} \neq S_{1}^{\{\neg, \wedge\}}+S_{1}^{\{\neg, \wedge\}} S_{1}^{\{\neg, \wedge\}}$ Solving (7) in $S$ we obtain two solutions, but one of them does not satisfy the condition $S(0)=S_{0}^{\{\neg, \wedge\}}=0$, hence we have proved our lemma.

By theorem 4 we have no even length formulae in the logic of nand, so to eliminate the zero values, we will use a bit different notation: $S_{n}^{\{\uparrow\}}$ denotes the number of formulae of length $2 n-1$. It is easy to check that in this logic $S_{1}^{\{\uparrow\}}=1$, moreover we set $S_{0}^{\{\uparrow\}}=0$. Let $\bar{S}(x)=\sum_{n=0}^{\infty} S_{n}^{\{\uparrow\}} x^{n}$ be the generating function of $S_{n}^{\{\uparrow\}}$.

\section{Lemma 3.}

$$
\bar{S}=\frac{1-\sqrt{1-4 x}}{2}
$$


Proof. $S_{n}^{\{\uparrow\}}=\sum_{i=1}^{n-1} S_{i}^{\{\uparrow\}} S_{n-i}^{\{\uparrow\}}$, because $2 n-1=2 i-1+2(n-i)-1+1$. Therefore $\bar{S}=\overline{S S}+x$, and solving this by using $\bar{S}(0)=S_{0}^{\{\uparrow\}}=0$ we proved our statement.

\section{$5 \quad$ Logic of negation and conjunction}

Remark 5. By Theorem 2 the relative frequencies of the logic of negation and disjunction can be derived easily from the relative frequencies of the logic of negation and conjunction.

In this section the set $\Gamma$ is $\{\neg, \wedge\}$. For the sake of brevity we will omit $\Gamma$, so write $\perp_{n}$ instead $\perp_{n}^{\{\neg, \wedge\}}$, etc.

Lemma 4. Let $B, D$ and $P$ be the generating functions of $B_{n}, D_{n}$ and $P_{n}$, respectively. Then

$$
\begin{aligned}
& B=\frac{1+x-\sqrt{1+3 x^{2}+2 x Y}}{2} \\
& D=\frac{1+x-Z}{2} \\
& P=\frac{2 x-Z+\sqrt{7 x^{2}-2 x Z+1+2 x Y-2 x \sqrt{1+3 x^{2}+2 x Y}}}{2}
\end{aligned}
$$

Here $Y=\sqrt{-(x+1)(3 x-1)}$ and $Z=\sqrt{1-x^{2}+2 x Y}$.

Proof. By (4),

$$
B_{n}=A_{n-1}+\sum_{i=1}^{n-1} B_{i} B_{n-i}
$$

that is we obtain a tautology or a n-formula of length $n-1$ by negating an antilogy or a p-formula of length $n-2$, or by conjugating two suitably long tautologies or n-formulae. Since $S_{n}=A_{n}+B_{n}=C_{n}+D_{n}$, thus $B=x(S-B)+B^{2}$ holds. Solving it and using that $B(0)=B_{0}=0$, we obtain (8). Similarly, we have $D=x(S-D)+D^{2}+x^{2}$, using that $D(0)=D_{0}=0$, we achieve (9). We obtain a p-formula of length $n-1$ either by negating an n-formula of length $n-2$, or by conjugating two suitably long p-formulae or a p-formula and a tautology. Hence,

$$
P_{n}=N_{n-1}+\sum_{i=1}^{n-1} P_{i} P_{n-i}+\sum_{i=1}^{n-1} \top_{i} P_{n-i}+\sum_{i=1}^{n-1} P_{i} \top_{n-i} .
$$

Since $\top_{j}=D_{j}-P_{j}$, and $N_{j}=C_{j}-\left(A_{j}-P_{j}\right)$, we have $P=x C+x A+x P+P^{2}+$ $2 P(D-P)+x^{2}$. Solving this equation and using that $P(0)=P_{0}=0$, we obtain (10).

Lemma 5. The singularity of $S, B, D$ and $P$ (defined by (6) and in lemma 4, respectively) with minimal modulus is $1 / 3$. 
Proof. By Remark 1, the functions $S, B, D$ and $P$ may have singularities when

$$
\begin{aligned}
1-2 x-3 x^{2} & =0 \\
1+3 x^{2}+2 x \sqrt{1-2 x-3 x^{2}} & =0 \\
1-x^{2}+2 x \sqrt{1-2 x-3 x^{2}} & =0
\end{aligned}
$$

or

$$
\begin{aligned}
7 x^{2} & -2 x \sqrt{1-x^{2}+2 x Y}+1+2 x \sqrt{1-2 x-3 x^{2}} \\
& -2 x \sqrt{1+3 x^{2}+2 x \sqrt{1-2 x-3 x^{2}}}=0
\end{aligned}
$$

The roots of (11) are -1 and $1 / 3$. The equation (12) has two solutions, which are approximately $-0.4110-0.3592 i$ and $-0.4110+0.3592 i$ having modulus $\approx 0.5458$. The equation (13) has also two solutions, which are -1 and $\approx-0.3733$. Finally the last equation has two solutions, which are approximately $0.6434+0.3378 i$ and $0.6434-0.3378 i$ having modulus $\approx 0.7267$.

Among the possible singularities $1 / 3$ has the smallest modulus, and $1 / 3$ is a singularity for all of $S, B, D$ and $P$.

Theorem 5. The relative frequency of tautologies, $p$-formulae, $n$-formulae and antilogies of length $n$ are asymptotically the following:

$$
\begin{array}{lcl}
\lim _{n \rightarrow \infty} \frac{P_{n}}{S_{n}}= & \frac{\sqrt{2}}{4}-\frac{12-3 \sqrt{2}-2 \sqrt{3}}{24 \sqrt{4-\sqrt{3}-\sqrt{2}}} & \approx 0.15995 \\
\lim _{n \rightarrow \infty} \frac{\top_{n}}{S_{n}}= & \frac{12-3 \sqrt{2}-2 \sqrt{3}}{24 \sqrt{4-\sqrt{3}-\sqrt{2}}} & \approx 0.19360 \\
\lim _{n \rightarrow \infty} \frac{N_{n}}{S_{n}}= & \frac{\sqrt{3}}{6}-\frac{12-3 \sqrt{2}-2 \sqrt{3}}{24 \sqrt{4-\sqrt{3}-\sqrt{2}}} & \approx 0.09507 \\
\lim _{n \rightarrow \infty} \frac{\perp_{n}}{S_{n}}= & \frac{12-3 \sqrt{2}-2 \sqrt{3}}{24}\left(2-\frac{1}{\sqrt{4-\sqrt{3}-\sqrt{2}}}\right) & \approx 0.55138
\end{array}
$$

Proof. By Lemma 5, the generating function $S(x)$ of the sequence $S_{n}$ has the minimal singularity $1 / 3$, and the function

$$
S(x / 3)=\frac{3-x-\sqrt{9-6 x-3 x^{2}}}{6}
$$

has an expansion in the form $\frac{1}{3}-\frac{\sqrt{3}}{3} \sqrt{1-x}+O(1-x)$. Again by Lemma 5 , the generating function $P(x)$ of the sequence $P_{n}$ has a singularity at $1 / 3$, which is the one with the least modulus, and the function $P(x / 3)$ has an expansion

$$
\frac{1+\sqrt{4-\sqrt{3}-\sqrt{2}}-\sqrt{2}}{3}+\left(\frac{4 \sqrt{3}-\sqrt{6}-2}{24 \sqrt{4-\sqrt{3}-\sqrt{2}}}-\frac{\sqrt{6}}{12}\right) \sqrt{1-x}+O(1-x)
$$


whence by Theorem 1 ,

$$
\lim _{n \rightarrow \infty} \frac{P_{n}}{S_{n}}=\frac{\sqrt{2}}{4}-\frac{12-3 \sqrt{2}-2 \sqrt{3}}{24 \sqrt{4-\sqrt{3}-\sqrt{2}}} .
$$

Similarly, the generating function $D(x)$ of the sequence $D_{n}$ has singularity $1 / 3$ with minimal modulus, and the function $D(x / 3)$ has an expansion $\frac{2-\sqrt{2}}{3}-$ $\frac{\sqrt{6}}{12} \sqrt{1-x}+O(1-x)$. Hence by the definition of $D_{n}$ and Theorem 1 ,

$$
\begin{aligned}
\lim _{n \rightarrow \infty} \frac{\top_{n}}{S_{n}}= & \lim _{n \rightarrow \infty} \frac{D_{n}-P_{n}}{S_{n}}=\lim _{n \rightarrow \infty} \frac{D_{n}}{S_{n}}-\lim _{n \rightarrow \infty} \frac{P_{n}}{S_{n}}= \\
& \frac{\sqrt{2}}{4}-\left(\frac{\sqrt{2}}{4}-\frac{12-3 \sqrt{2}-2 \sqrt{3}}{24 \sqrt{4-\sqrt{3}-\sqrt{2}}}\right)= \\
& \frac{12-3 \sqrt{2}-2 \sqrt{3}}{24 \sqrt{4-\sqrt{3}-\sqrt{2}}} .
\end{aligned}
$$

Furthermore, the generating function $B(x)$ of the sequence $B_{n}$ has singularity $1 / 3$ with minimal modulus, and the function $B(x / 3)$ has an expansion $\frac{2-\sqrt{3}}{3}-$ $\frac{1}{6} \sqrt{1-x}+O(1-x)$. Hence by the definition of $B_{n}$ and Theorem 1 ,

$$
\begin{aligned}
\lim _{n \rightarrow \infty} \frac{N_{n}}{S_{n}}= & \lim _{n \rightarrow \infty} \frac{B_{n}-\top_{n}}{S_{n}}=\lim _{n \rightarrow \infty} \frac{B_{n}}{S_{n}}-\lim _{n \rightarrow \infty} \frac{\top_{n}}{S_{n}}= \\
& \frac{\sqrt{3}}{6}-\frac{12-3 \sqrt{2}-2 \sqrt{3}}{24 \sqrt{4-\sqrt{3}-\sqrt{2}}} .
\end{aligned}
$$

Since $S_{n}=A_{n}+B_{n}$, thus

$$
\begin{aligned}
\lim _{n \rightarrow \infty} \frac{\perp_{n}}{S_{n}}= & \lim _{n \rightarrow \infty} \frac{A_{n}-P_{n}}{S_{n}}=\lim _{n \rightarrow \infty} \frac{S_{n}-B_{n}-P_{n}}{S_{n}}= \\
& \lim _{n \rightarrow \infty} \frac{S_{n}}{S_{n}}-\lim _{n \rightarrow \infty} \frac{B_{n}}{S_{n}}-\lim _{n \rightarrow \infty} \frac{P_{n}}{S_{n}}= \\
& 1-\frac{\sqrt{3}}{6}-\left(\frac{\sqrt{2}}{4}-\frac{12-3 \sqrt{2}-2 \sqrt{3}}{24 \sqrt{4-\sqrt{3}-\sqrt{2}}}\right)= \\
& \frac{12-3 \sqrt{2}-2 \sqrt{3}}{24}\left(2-\frac{1}{\sqrt{4-\sqrt{3}-\sqrt{2}}}\right) .
\end{aligned}
$$

\section{$6 \quad$ Logic of nand}

Remark 6. By Theorem 3 the relative frequencies of the logic of nor can be derived easily from the relative frequencies of the logic of nand. 
In this section the set $\Gamma$ is $\{\uparrow\}$. For the sake of brevity we omit $\Gamma$, hence we write $\perp_{n}$ instead $\perp_{n}^{\{\uparrow\}}$, etc.

Lemma 6. Let $A, C$ and $P$ be the generating functions of $A_{n}, C_{n}$ and $P_{n}$, respectively. Then

$$
\begin{aligned}
A & =1-\frac{1}{2} Y-\frac{1}{2} Z_{2} \\
C & =1-\frac{1}{2} Y-\frac{1}{2} Z_{1} \\
P & =-1+\frac{1}{2} Z_{1}+\frac{1}{2} \sqrt{7-2 Z_{1}-2 Y-2 Z_{2}}
\end{aligned}
$$

where $Y=\sqrt{1-4 x}, Z_{1}=\sqrt{3-2 Y}$ and $Z_{2}=\sqrt{3-2 Y-4 x}$.

Proof. By (4),

$$
A_{n}=\sum_{i=1}^{n-1}\left(S_{i}-A_{i}\right)\left(S_{n-i}-A_{n-i}\right) \text { and } C_{n}=\sum_{i=1}^{n-1}\left(S_{i}-C_{i}\right)\left(S_{n-i}-C_{n-i}\right)
$$

For the corresponding generating functions we have $A=(\bar{S}-A)(\bar{S}-A)+x$ and $C=(\bar{S}-C)(\bar{S}-C)$. Solving these equations we get (14) and (15). By (3) and the definition of $A, B, C$ and $D$, we have

$$
\begin{aligned}
P_{n}= & \sum_{i=1}^{n-1} N_{i}\left(2 \top_{n-i}+N_{n-i}\right)=\sum_{i=1}^{n-1} N_{i}\left(2 B_{n-i}-N_{n-i}\right)= \\
& \left.\sum_{i=1}^{n-1}\left(C_{i}-A_{i}+P_{i}\right)\left(2 S_{n-i}-A_{n-i}-C_{n-i}-P_{n-i}\right)\right) .
\end{aligned}
$$

From this we get $P=(C-A+P)(2 \bar{S}-A-C-P)+x$. Solving this equation we get (16).

Lemma 7. The singularity of $\bar{S}, A, C$ and $P$ (defined in lemma 6 ) with minimal modulus is $1 / 4$.

Proof. The functions $\bar{S}, A, C$ and $P$ may have singularities when

$$
\begin{array}{r}
1-4 x=0 \\
3-2 \sqrt{1-4 x}=0 \\
3-2 \sqrt{1-4 x}-4 x=0
\end{array}
$$

or

$$
7-2 \sqrt{3-2 \sqrt{1-4 x}}-2 \sqrt{1-4 x}-2 \sqrt{3-2 \sqrt{1-4 x}-4 x}=0 .
$$

The root of (17) is $1 / 4$. The equation (18) has solution $-5 / 16$, and the equation (19) has two solutions: $1 / 4-1 / 2 i$ and $1 / 4+1 / 2 i$. Finally the last equation has two 
solutions, which are approximately $-0.41823-0.48428 i$ and $-0.41823+0.48428 i$ having modulus $\approx 0.63987$.

Among the possible singularities $1 / 4$ has the smallest modulus, and $1 / 4$ is a singularity for all of $\bar{S}, A, C$ and $P$.

Theorem 6. The relative frequency of tautologies, $p$-formulae, $n$-formulae and antilogies of length $n$ are asymptotically the following:

$$
\begin{array}{lcl}
\lim _{n \rightarrow \infty} \frac{P_{n}}{S_{n}}= & \frac{\sqrt{3}}{3}-\frac{2 \sqrt{3}-6+3 \sqrt{2}}{6 \sqrt{7-2 \sqrt{3}-2 \sqrt{2}}} & \approx 0.23916 \\
\lim _{n \rightarrow \infty} \frac{\top_{n}}{S_{n}}= & \frac{2 \sqrt{3}-6+3 \sqrt{2}}{6 \sqrt{7-2 \sqrt{3}-2 \sqrt{2}}} & \approx 0.33819 \\
\lim _{n \rightarrow \infty} \frac{N_{n}}{S_{n}}= & \frac{\sqrt{2}}{2}+\frac{2 \sqrt{3}-6+3 \sqrt{2}}{6 \sqrt{7-2 \sqrt{3}-2 \sqrt{2}}} & \approx 0.36892 \\
\lim _{n \rightarrow \infty} \frac{\perp_{n}}{S_{n}}= & \frac{6-2 \sqrt{3}-3 \sqrt{2}}{6}\left(1+\frac{1}{\sqrt{7-2 \sqrt{3}-2 \sqrt{2}}}\right) & \approx 0.05373
\end{array}
$$

Proof. By Lemma 7, the generating function $\bar{S}(x)$ of the sequence $S_{n}$ has the only singularity $1 / 4$, and the function

$$
\bar{S}(x / 4)=\frac{1-\sqrt{1-x}}{2}
$$

has an expansion in the form $\frac{1}{2}-\frac{1}{2} \sqrt{1-x}$. Again by Lemma 7 , the generating function $P(x)$ of the sequence $P_{n}$ has a singularity at $1 / 4$, which is the one with the least modulus, and the function $P(x / 4)$ has an expansion $\frac{-2+\sqrt{3}+\sqrt{7-2 \sqrt{3}-2 \sqrt{2}}}{2}+$ $\left(-\frac{\sqrt{3}}{6}+\frac{2 \sqrt{3}-6+3 \sqrt{2}}{12 \sqrt{7-2 \sqrt{3}-2 \sqrt{2}}}\right) \sqrt{1-x}+O(1-x)$, whence by Theorem 1 ,

$$
\lim _{n \rightarrow \infty} \frac{P_{n}}{S_{n}}=\frac{\sqrt{3}}{3}-\frac{2 \sqrt{3}-6+3 \sqrt{2}}{6 \sqrt{7-2 \sqrt{3}-2 \sqrt{2}}} .
$$

Similarly, the generating function $A(x)$ of the sequence $A_{n}$ has singularity $1 / 4$ with minimal modulus, and the function $A(x / 4)$ has an expansion $1-\frac{\sqrt{2}}{2}+$ $\frac{-2+\sqrt{2}}{4} \sqrt{1-x}+O(1-x)$. Hence by the definition of $A_{n}$ and Theorem 1 ,

$$
\begin{aligned}
\lim _{n \rightarrow \infty} \frac{\perp_{n}}{S_{n}}= & \lim _{n \rightarrow \infty} \frac{A_{n}-P_{n}}{S_{n}}=1-\frac{\sqrt{2}}{2}-\left(\frac{\sqrt{3}}{3}-\frac{2 \sqrt{3}-6+3 \sqrt{2}}{6 \sqrt{7-2 \sqrt{3}-2 \sqrt{2}}}\right)= \\
& \frac{6-2 \sqrt{3}-3 \sqrt{2}}{6}\left(1+\frac{1}{\sqrt{7-2 \sqrt{3}-2 \sqrt{2}}}\right) .
\end{aligned}
$$

Furthermore, the generating function $C(x)$ of the sequence $C_{n}$ has singularity $1 / 4$ with minimal modulus, and the function $C(x / 4)$ has an expansion $1-\frac{\sqrt{3}}{2}+$ 
$\frac{-3+\sqrt{3}}{6} \sqrt{1-x}+O(1-x)$. Hence by the definition of $C_{n}$ and Theorem 1 ,

$$
\lim _{n \rightarrow \infty} \frac{N_{n}}{S_{n}}=\lim _{n \rightarrow \infty} \frac{C_{n}-\perp_{n}}{S_{n}}=\frac{\sqrt{2}}{2}+\frac{2 \sqrt{3}-6+3 \sqrt{2}}{6 \sqrt{7-2 \sqrt{3}-2 \sqrt{2}}} .
$$

Since $S_{n}=C_{n}+D_{n}$, thus

$$
\lim _{n \rightarrow \infty} \frac{\top_{n}}{S_{n}}=\lim _{n \rightarrow \infty} \frac{D_{n}-P_{n}}{S_{n}}=\frac{2 \sqrt{3}-6+3 \sqrt{2}}{6 \sqrt{7-2 \sqrt{3}-2 \sqrt{2}}} .
$$

\section{Summary}

The logic of negation and equivalence is not a known logic, because in the general case we cannot express any truth-value function with the help of negation and equivalence. But if we have only one propositional variable, we can construct formulae equivalent with $p, \neg p, \top$ and $\perp$. We leave it to the reader to check that a formula of this language is a tautology if and only if it contains even number of negations and odd number of equivalences. (Hint. Use the following rewrite rules: $(\neg \varphi \equiv \psi) \sim \neg(\varphi \equiv \psi),((\varphi \equiv \psi) \equiv \chi) \sim \varphi \equiv \psi \equiv \chi, p \equiv p \equiv p \sim p$, $\varphi \equiv \psi \sim \psi \equiv \varphi$ and double negation.)

From this it follows that the length of each tautology is even. This is the reason why $\top_{n} / S_{n}$ has no limit. We will discuss this logic in a separate article.

Following the method described in the proof of Theorem 1, we were able compute the relative frequency of tautologies, $\mathrm{p}$-formulae, $\mathrm{n}$-formulae and antilogies in several logics, determined by different combinations of connectives. The table below summarizes the numerical results of the calculations of different ratios.

\begin{tabular}{ccccc}
\hline$\Gamma$ & \multicolumn{4}{c}{ Approximation of proportion of } \\
& $p$-formulae & antilogies & $n$-formulae & tautologies \\
\hline$\uparrow$ & $23.916 \%$ & $5.373 \%$ & $36.892 \%$ & $33.819 \%$ \\
$\downarrow$ & $23.916 \%$ & $33.819 \%$ & $36.892 \%$ & $5.373 \%$ \\
$\neg \wedge$ & $15.995 \%$ & $55.138 \%$ & $9.507 \%$ & $19.360 \%$ \\
$\neg \vee$ & $15.995 \%$ & $19.360 \%$ & $9.507 \%$ & $55.138 \%$ \\
$\neg \wedge \vee$ & $32.498 \%$ & $26.081 \%$ & $15.341 \%$ & $26.081 \%$ \\
$\neg \wedge \supset$ & $27.803 \%$ & $19.986 \%$ & $15.906 \%$ & $36.305 \%$ \\
$\neg \wedge \supset \equiv$ & $27.804 \%$ & $20.681 \%$ & $17.787 \%$ & $33.729 \%$ \\
\hline
\end{tabular}

\section{References}

[1] Belnap, Jr., Nuel D. A useful four-valued logic. In Modern uses of multiplevalued logic (Fifth Internat. Sympos., Indiana Univ., Bloomington, Ind., 1975), pages 5-37. Episteme, Vol. 2. Reidel, Dordrecht, 1977. 
[2] Harris, Mitchell A. Counting satisfiable k-cnf formulas. In Walsh, Toby, editor, Principles and Practice of Constraint Programming - CP 2001, 7th International Conference, CP 2001, Paphos, Cyprus, November 26 - December 1, 2001, Proceedings, volume 2239 of Lecture Notes in Computer Science, page 765. Springer, 2001.

[3] Kostrzycka, Zofia. On the density of truth in Grzegorczyk's modal logic. Bull. Sect. Logic Univ. Eódź, 33(2):107-120, 2004.

[4] Kostrzycka, Zofia. Density of truth in modal logics. In Fourth Colloquium on Mathematics and Computer Science Algorithms, Trees, Combinatorics and Probabilities, Proceedings, pages 161-170. www.dmtcs.org, 2006.

[5] Kostrzycka, Zofia and Zaionc, Marek. Statistics of intuitionistic versus classical logics. Studia Logica, 76(3):307-328, 2004.

[6] Moczurad, M., Tyszkiewicz, J., and Zaionc, M. Statistical properties of simple types. Math. Structures Comput. Sci., 10(5):575-594, 2000.

[7] Soza, Grzegorz. Asymptotic density as a method of expressing quantitative relations in intuitionistic logic. Rep. Math. Logic, (36):71-93, 2002.

[8] Szegő, Gábor. Orthogonal polynomials, volume XXIII of AMS Colloquium Publications. AMS, 1939.

[9] Wilf, Herbert S. generatingfunctionology. A K Peters, third edition, 2005.

[10] Zaionc, Marek. On the asymptotic density of tautologies in logic of implication and negation. Rep. Math. Logic, (39):67-87, 2005.

[11] Zaionc, Marek. Probability distribution for simple tautologies. Theor. Comput. Sci., 355(2):243-260, 2006. 